hep-th/9611125

RU-96-102

\title{
D-branes and Near Extremal Black Holes at Low Energies
}

\author{
Juan Maldacena1 \\ Department of Physics and Astronomy, Rutgers University, Piscataway, NJ 08855, USA
}

\begin{abstract}
It has been observed recently that many properties of some near extremal black holes can be described in terms of bound states of D-branes. Using a non-renormalization theorem we argue that the D-brane description is the correct quantum gravity description of the black hole at low energies. The low energy theory includes the black hole degrees of freedom that account for the entropy and describes also Hawking radiation. The description is unitary and there seems to be no information loss at low energies.
\end{abstract}

\footnotetext{
1 malda@physics.rutgers.edu
} 


\section{Introduction}

Recently [1] the entropy of extremal black holes in string theory was calculated by counting the number bound states of D-branes. The D-brane description corresponds to the weak coupling limit while the black hole description corresponds to strong coupling. In the first case the gravitational radius of the configuration is smaller than the string scale while it is bigger than the string scale for the latter. Extremal black holes are supersymmetric BPS solutions. Supersymmetric nonrenormalization arguments ensure that we can do the counting of states at small coupling and then extrapolate the result to the strong coupling domain. This ensures that the D-brane counting agrees with the classical area law for the black hole entropy [1].

While this explains the agreement found for extremal BPS solutions [1],22, 3, [4] it has not been clear why D-brane calculations for near extremal black holes also agree with black holes. The agreement includes entropy counting [5, 6, 67,8, 9,9] as well as more detailed dynamical properties such as absorption cross sections and Hawking radiation [10,11, 12, 13, 14, 15, .

Here we give a rationale for this agreement for a class of near extremal five dimensional black holes (in the so called dilute gas region). The excitations of the D-brane system at low energies are described in terms of a moduli space approximation. Using a nonrenormalization theorem we argue that this low energy theory receives no corrections when we increase the coupling and we go from the D-brane region into the black hole region. Therefore the same moduli space describes the low energy dynamics in the black hole region. We then argue that the energy of the excitations accounting for the entropy and Hawking radiation are low enough to be described within the low energy field theory. In order to do this we estimate the size of the corrections to the low energy theory, we estimate this on the weakly coupled side and we see that extending this criterion to the strong coupling region gives a sensible picture.

We start in section 2 by describing the regime of interest, the type of black holes considered as well as the low energy condition. In section 3 we describe the low energy D-brane theory and argue that it can be extrapolated to strong coupling, provided the energy is low enough, we also give the condition that the energy has to satisfy. In section 4 we explain why things calculated in the two regimes should agree. In section 5 we study the possibility of D-brane emission. In section 6 we argue that these results imply that the dynamics for these black holes is unitary at low energies. 


\section{Low energy field theory}

We start with type IIB string theory compactified on $T^{5}=T^{4} \times S^{1}$. We consider five dimensional black holes (or six dimensional long strings) parameterized by the four classical parameters $r_{0}, r_{n}, r_{1}, r_{5}$, the four parameters correspond to three charges and the mass. The explicit solution is written in [16] and we follow the conventions there. The charges correspond to a system of $Q_{5}$ D-fivebranes wrapped on $T^{5}, Q_{1}$ D-onebranes wrapped on $S_{1}$ and momentum $P=n / R$ along $S^{1}$.

We consider the dilute gas region defined by 13

$$
r_{0}, r_{n} \ll r_{1}, r_{5}
$$

for reasons that will become clearer later. In most of the discussion we take the size of the $T^{4}$ to be small, of order $V_{4} \sim \alpha^{\prime 2}$ and $S^{1}$ very long (we will discuss what changes if $S^{1}$ is small later on) and we take $\alpha^{\prime}=1$ (all lengths are measured in units of $\sqrt{\alpha^{\prime}}$ ). We also take $Q_{1} \sim Q_{5} \sim Q$, all these approximations are done for simplicity and clarity in the argument and it is straightforward to extend the arguments for more general values of $V_{4}$ and $Q_{1} \neq Q_{5}$. Under these conditions $r_{1} \sim r_{5}$. The typical gravitational radius of the black hole is $r_{g}^{2}=\max \left\{r_{1}^{2}, r_{5}^{2}\right\} \sim g Q$. The gravitational radius is defined by the condition that the redshift between a static observer and the asymptotic observer becomes of order one.

In all our discussion the coupling $g$ is small $g \ll 1$ so that closed string effects are small. However the effective open string coupling is $g Q$ since it is like a large $N$ gauge theorye $2(N=Q)$. When the coupling is weak $g Q \ll 1$ then we are in the domain of validity of the D-brane perturbation theory. If $g Q \gg 1$ we say that the coupling is strong and we are in the semiclassical black hole domain. Note that this definition of strong coupling is not the strong coupling region $g \gg 1$ which is present in usual discussions on string dualities. Here we have strong coupling because of the large number $Q$ of branes.

We also consider the low energy field theory, the theory were the energies of all particles satisfy

$$
\omega^{2} r_{g}^{2} \ll 1 \quad \text { or } \quad \omega^{2} g Q \ll 1
$$

For example, in a scattering process the energies of the particles measured at infinity satisfy (2.2). In this limit the Compton wavelength of the particle is much bigger than the size of

2 The open string coupling is $g_{\text {open }} \sim \sqrt{g}$. 
the black hole, so the black hole appears effectively as a pointlike system from the point of view of the low energy theory on the bulk. Note that energies are low with respect to $1 / r_{g}$ which can itself be very low, for an astronomical size black hole this energy is extremely low, in particular much smaller than the string scale, the compactification scale and other microscopic scales in the problem.

In the low energy black hole region one can do calculations using the method of quantum fields on a fixed classical background, this is the semiclassical domain, it is the domain in which Hawking radiation occurs, for near extremal black holes the wavelength of Hawking radiation is much bigger than the gravitational size of the black hole, $1 / T_{H} \gg r_{g}$. If $1 \ll r_{0}^{2}+r_{n}^{2}, r_{1}^{2}, r_{5}^{2}$ these calculations do not receive any $\alpha^{\prime}$ corrections 3 . One can compute Hawking radiation in this way, absorption cross sections, etc. The traditional semiclassical view [17] [18] is that in this case we can only have a thermal description of the system, the emitted particles do not know about the microscopic state of the black hole.

There are however things that we cannot do in this low energy domain, we cannot measure the local geometry, since waves have wavelengths much greater than the gravitational radius, the observer at infinity cannot measure the precise shape of the metric outside the horizon. His measuring rod is longer than the black hole. For him the black hole is as a pointlike system that can absorb energy and radiate it back thermally. It should be noted however that the absorption cross section depends on some features of the geometry, so it is in some sense a measure of the geometry, but not detailed enough to sense the precise form of the metric.

3 If $r_{0} \gg 1$ it is easy to see that there is a smooth horizon, of size bigger than $\alpha^{\prime}$, however we could also have a smooth horizon in the extremal limit, $r_{0}=0$, as long as $r_{n} \gg 1$ (in other words, as long as we have three large charges $\left.\left(n, Q_{1}, Q_{5}\right)\right)$. 


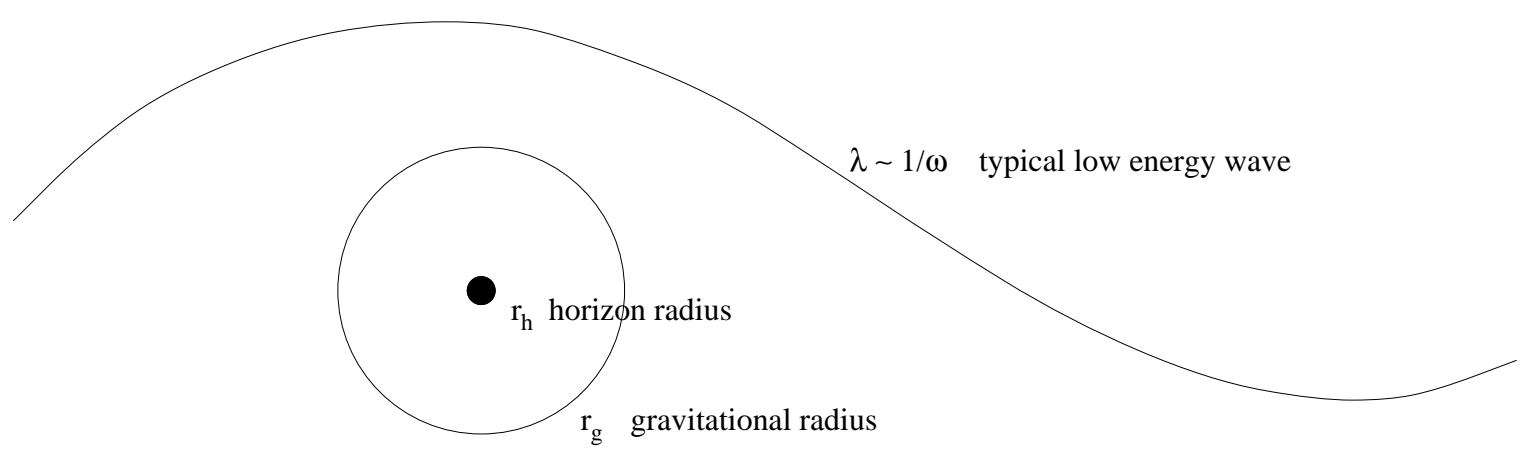

FIGURE 1: Various scales in the problem, with $r_{0}, r_{n} \ll r_{1}, r_{5} \ll \lambda$. The sizes of the circles give an idea of the areas of the 3 -spheres and $\lambda$ is the typical wavelength of the particles we scatter.

\section{D-brane low energy theory, Moduli space approximation.}

We will now concentrate on the open string sector of the theory describing the excitations of the D-branes. This sector becomes strongly coupled in the black hole region. This theory is a $(1+1)$ dimensional field theory with $(4,4)$ supersymmetry since this is the supersymmetry left unbroken by the extremal D-branes (1D $+5 \mathrm{D}$ branes). This supersymmetry is similar to $\mathrm{N}=2$ in $\mathrm{D}=4$. These theories have vector multiplets and hypermultiplets. In two dimensions the vector multiplet and hypermultiplet seem very similar, both have four physical scalar components. The distinction between them is that they have different transformation properties under $R$ symmetries. This was discussed in the context of three dimensional theories in [19] where the same problem appeared. To understand this it is useful to think of this theory as the dimensional reduction of a six dimensional $N=1$ theory. In six dimensions there is a $\mathrm{SU}(2)_{R}$ symmetry, the vector multiplet has no scalar components and its bosonic components are trivial under the $\mathrm{SU}(2)_{R}$. On the other hand the hypermultiplet has four scalar components transforming as the 2 of $\mathrm{SU}(2)_{R}$. When we reduce to two dimensions we have an extra $\mathrm{SO}(4) \sim \mathrm{SU}(2)_{\tilde{L}} \times \mathrm{SU}(2)_{\tilde{R}} \mathrm{R}$-symmetry, again the vector and hypermultiplets will transform differently under these R-symmetries and that is what distinguishes them. It is interesting also that the two $\mathrm{SU}(2)$ factors coming from $\mathrm{SO}(4)$ are correlated with the chirality in the $1+1$ dimensional theory, $\tilde{L}$ and $\tilde{R}$ denote also left and right moving. The vector multiplets are related to separation of the branes in the extended four spatial dimensions and the hypermultiplets correspond to "dissolving" the onebranes inside the fivebrane [20]. This $\mathrm{SO}(4)$ symmetry of the gauge theory corresponds the $\mathrm{SO}(4)$ rotational symmetry of the five dimensional black hole [2]. 
When we go to low energies we will keep only the massless excitations and terms in the Lagrangian which are at most quadratic in the velocities. The D-brane low energy theory consists $4 Q_{1} Q_{5}$ massless fields parameterizing the moduli space of the bound state of 1 branes and 5 branes. The moduli space is topologically

$$
\mathcal{M}=\left(T^{4}\right)^{Q_{1} Q_{5}} / S\left(Q_{1} Q_{5}\right)
$$

where $S(m)$ is the permutation group of $m$ elements. This moduli space was obtained by duality arguments (by Vafa [21]) and it was later shown in [22] [23] that this gives a microscopic counting of BPS states with charges $n, Q_{1}, Q_{5}$ which is fully U-duality invariant. Summarizing, the situation is that we know by indirect arguments that the moduli space should be (3.1), at least topologically. In principle one could calculate the metric on this moduli space in the weakly coupled D-brane theory.

As in four [24] and three [25] dimensions it is possible to prove that supersymmetry implies that there are no couplings between vectors and neutral hypermultiplets. A simple way to see this the followingt, first we choose two left moving and two rightmoving supercharges out of the $(4,4)$ available and we realize explicitly a $(2,2)$ supersymmetry by using $(2,2)$ superfields. In terms of $(2,2)$ superfields the hypermultiplet decomposes into a pair of chiral multiplets $\phi_{h}$ and the vector decomposes into a chiral multiplet $\phi_{v}$ and a twisted chiral multiplet $\chi$ [26]. The general $(2,2)$ Lagrangian for chiral and twisted chiral fields was considered in [26]. It is determined by a single function $K\left(\phi_{p}, \bar{\phi}_{q}, \chi_{a}, \bar{\chi}_{b}\right)$ which gives the metric and antisymmetric tensor field (B-field) of a non-linear sigma model

$$
\begin{aligned}
G_{p \bar{q}} & =\partial_{p} \partial_{\bar{q}} K, & G_{a \bar{b}} & =-\partial_{a} \partial_{\bar{b}} K \\
B_{p \bar{b}} & =\partial_{p} \partial_{\bar{b}} K, & B_{\bar{q} a} & =\partial_{\bar{q}} \partial_{a} K
\end{aligned}
$$

and the rest vanishes, including the metric components mixing the chiral and twisted chiral multiplets $G_{p \bar{b}}=0$ and $B_{p \bar{q}}=B_{a \bar{b}}=0$. If we now perform a $\mathrm{SU}(2)_{\tilde{L}}$ rotation on the system we can define new $\left(2^{\prime}, 2^{\prime}\right)$ charges so that the chiral multiplets coming from the vector multiplet become twisted chiral and vice versa, $\phi_{v}^{\prime}=\chi, \quad \chi^{\prime}=\phi_{v}$, the chiral multiplets coming from the hypermultiplets $\phi_{h}$ stay as chiral multiplets. Combining the constraints of $(2,2)$ invariance with $\left(2^{\prime}, 2^{\prime}\right)$ invariance we conclude that the metric and Bfield components mixing the hypermultiplets with the vector multiplets vanish, $G_{\phi_{h} \phi_{v}}=$ $G_{\phi_{h} \chi}=B_{\phi_{h} \phi_{v}}=B_{\phi_{h} \chi}=0$. Using eqn. (3.2) we see that the sigma model factorizes

4 I thank D. Kabat for pointing out an error in my previous argument. 
$K=K\left(\phi_{h}, \bar{\phi}_{h}\right)+K\left(\phi_{v}, \bar{\phi}_{v}, \chi, \bar{\chi}\right)$. The hypermultiplet metric is then hyperkähler since the sigma model has $(4,4)$ supersymmetry and it has no torsion (B-field) [27]. The vector multiplet moduli space corresponds to the models studied in [26] and it is a generalized "hyperkähler" manifold, which in some cases can be reduced, via a duality transformation, to a usual hyperkähler manifold [26]. In any case, the conclusion is that the hypermultiplets are decoupled from the vector multiplets 0 .

We are interested in the hypermultiplet moduli space since it parameterizes the space of possible bound state configurations [1] [28]. Following the ideas in [29] we regard the coupling constant as a background field, which should then be a vector multiplet since it appears in front of the gauge kinetic term, an interaction that would be forbidden if it were a hypermultiplet. This implies that there are no corrections, perturbative or non perturbative, to the hypermultiplet moduli space. This implies that the hyperkähler metric, once we calculate it, is not renormalized when we increase the coupling.

In two dimensions we also have to worry about the fact that vacuum expectation values are not well defined for massless fields. It is more accurate to speak about the resulting conformal field theory rather than the moduli space itself. It is a conformal field theory because a hyperkähler metric is Ricci flat [27]. The statement would be that the conformal field theory can be extrapolated from weak to strong coupling. However there is another related problem which is that the branches on the moduli space are not so well separated. There is a nonvanishing probability for the system to wander into the vector moduli space, which corresponds physically to the emission of D-branes, the scalars of the vector multiplet correspond to separating the brane in the extended $R^{4}$ spatial dimensions. We will argue in sec. 6 that this process is highly suppressed for entropy reasons. Similar problems appear when non-renormalization theorems are applied to the quantum mechanics of D0-branes 30] 31.

As a aside, notice that there are indeed corrections to the vector moduli space, for example if a one brane is far from the fivebranes then the moduli space is classically flat but there is a one loop correction coming from integrating out the massive $(1,5)$ strings that gives the $g Q / r^{2}$ correction to the metric in moduli space [32]. This also shows that the coupling constant is indeed in a vector multiplet, otherwise it could not have affected the vector multiplet moduli space.

5 They are decoupled locally but there could be some gobal identifications, which can usually be seen classically and will not affect our later argument. I thank N. Seiberg for pointing this out to me. 
Note that the "D-brane theory" that has been applied to compute the entropy [5], and scattering cross sections [11], [13], was precisely this moduli space approximation to the motion of the D-branes since only the massless excitations on the branes were taken into account. So it is this moduli space approximation that has been observed, by direct calculation, to agree with the semiclassical results at strong coupling.

The conclusion is then that at low enough energies the excitations of the system are correctly described by this moduli space approximation, even for strong coupling!. Now the question is: what energies are "low enough"?

First let us estimate, in the weak coupling theory, what the mass of the least massive states is. One appealing picture is to think of the one brane charge as carried by instantons on the fivebrane gauge theory [20]. However this parameterization is physically reasonable only when $Q_{1} \ll Q_{5}$ (more precisely $r_{1}^{2} \ll r_{5}^{2}$ ) otherwise the total energy in the instantons in comparable to the energy of the fivebranes and the fivebrane might bend or deform where there are many instantons. In other words, higher order terms in a Dirac-BornInfeld-type action of the fivebrane might be important. In the case of $Q_{1} \sim Q_{5}$ it seems more reasonable to consider a set of two intersecting three branes (intersecting along the $\left.S^{1}\right)$. Then the massless degrees of freedom are somehow associated to the $Q_{1} Q_{5}$ intersection lines. The transverse space of each set of three branes is a two torus (say of size $\alpha^{\prime}$ ). The three branes look like points on this two torus. If we assume that the $Q$ three branes are uniformly distributed we find that the distance between one and the nearest neighbor is typically $r^{2} \sim 1 / Q$, so that $m^{2} \sim 1 / Q$.

Corrections due to the massive modes will go like

$$
g \frac{\omega^{2}}{m^{2}}
$$

This implies that the corrections due to the lightest massive mode are proportional to

$$
g Q \omega^{2} \ll 1
$$

which is small in the regime defined by (2.2). There are also some other possibly light modes like D-strings going between two different threebranes, which would have a mass $m^{2} \sim \frac{1}{g Q}$ and an interaction strength of order one, giving corrections proportional to (3.4) again. There are points in the moduli space where some states could become light, for example if two threebranes come close to each other. This seems to affect a small fraction of the hypermultiplets ( $Q$ of them vs. a total of $Q^{2}$ ) therefore it will result in a small 
correction. Since there is a large number of massive states there are large $N(=Q)$ effects going like

$$
g Q \frac{\omega^{2}}{\bar{m}^{2}}
$$

where $\bar{m}$ is the average mass and $g Q$ is the effective large $N(=Q)$ coupling. But $\bar{m}^{2} \sim 1$ since the typical distance between any two threebranes is of the order of the compactification volume, so that we get (2.2) again. Presumably all other effects we could imagine would also be proportional to (3.4).

In the case that the radius of the circle is small the low energy theory corresponds to a $1+1$ dimensional field theory whose target space is the moduli space (3.1) but now on small circle. The fact that we divided out by the permutation group enables us to have twisted sectors in the low energy conformal field theory which correspond to long multiply wound "fractional" strings [23]. These twisted sectors support excitations whose energy gap is much smaller than $1 / R\left(R\right.$ is the radius of $\left.S_{1}\right)$. The gap actually becomes $\frac{1}{R Q_{1} Q_{5}}$ which is much smaller than $T_{L}, T_{R}$ in the limit of large charges [33.

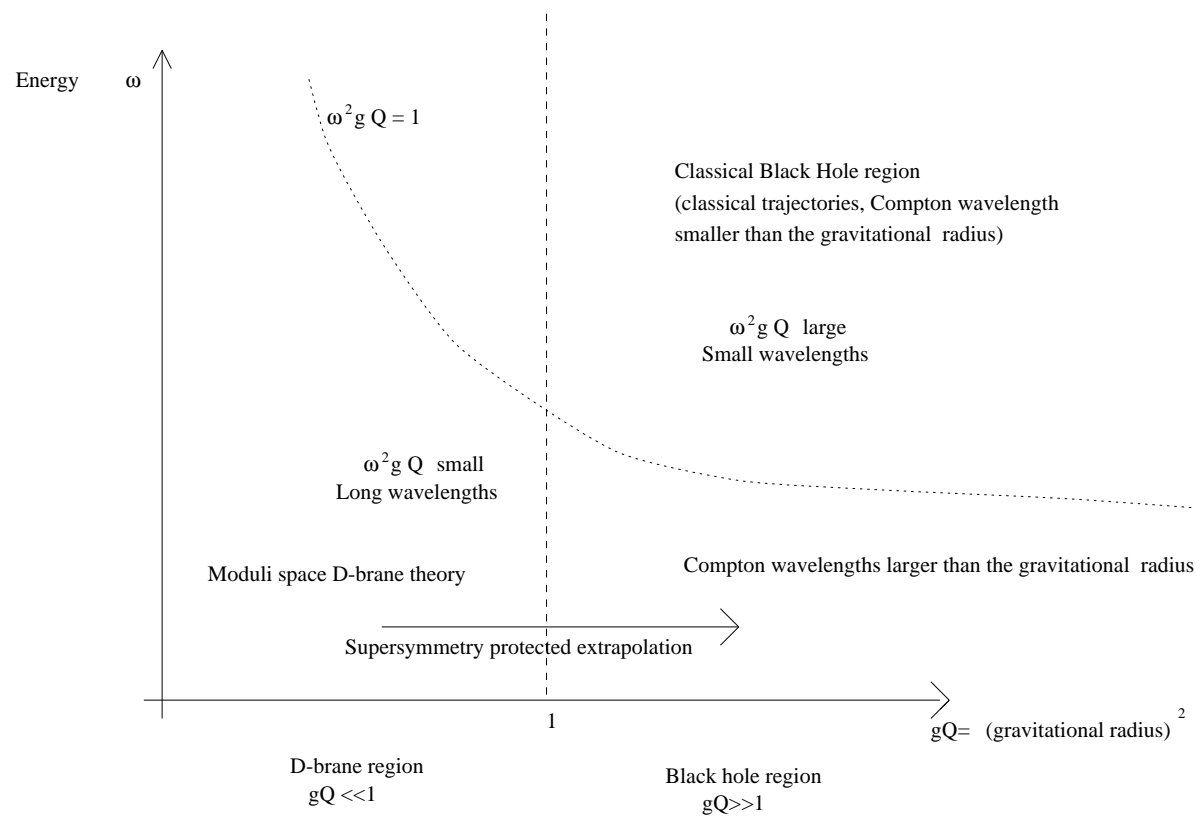

FIGURE 2: Different regions in the space of parameters of a near extremal configuration. D-brane results can be extrapolated for low energies.

\section{D-brane vs. Black hole computations}

We saw in the previous section that the moduli space metric for the system of one 
and fivebranes is not changed as we make the coupling strong. This non-renormalization theorem ensures that there are some low energy processes that can be calculated in the strong coupling regime (the black hole regime). The entropy of the system will be accurately given by the moduli space approximation if the typical energy of the massless modes, which is proportional to $T_{L}, T_{R}$, satisfies (2.2). This is indeed the case if $r_{0}, r_{n} \ll r_{1}, r_{5}$ since the temperatures are bounded by $T_{L, R} \leq \frac{\sqrt{r_{0}^{2}+r_{n}^{2}}}{r_{1} r_{5}} \ll 1 / r_{g}$ [5]. So we conclude that the entropy is accurately given by the D-brane moduli space approximation, provided we are in the dilute gas region (2.1).

Now let us turn to the scattering processes considered in [11], [13]. The scalar considered there was an internal component of the metric $h_{i j}$ of the four torus. Since this metric appears in the moduli space metric of the low energy D-brane theory, we conclude that its coupling to the massless degrees of freedom is not renormalized.

The calculations [15] that probe the higher order terms in the Nambu action might also be understood by using this line of argument. The moduli space (3.1) seems to imply that the excitations of the system are "fractional" strings, this is indeed true for BPS states [23]. It seems natural that these strings should couple to the background metric with the Nambu action. This deserves a more careful analysis.

\section{Black hole fragmentation 6 .}

One of the possible decay modes of a black hole is by emission of charged particles, by which the black hole loses its charge, in some sense it fragments into the elementary constituents. In principle it can emit KK momentum, one brane winding charge and fivebrane charge, the first one can be described in the D-brane moduli space approximation described above [12], [13] and the last two correspond to some D-brane leaving the system. If we have a near extremal black hole which carries three charges large $n, Q_{1}, Q_{5}$ then there is an entropic suppression factor for the emission of charged particles. For example if it emits one unit of KK momentum, then the change in the extremal black hole entropy is $\delta S=\pi \sqrt{Q_{1} Q_{5} / n}$. The emission amplitude therefore has a phase space suppression factor $e^{-\delta S}$. If all charges are large then $\delta S$ is very large. This is independent of whether we are in the dilute gas approximation or not, here the question is whether the quantized values of the charges are large or not.

6 Many of the remarks in this section originated in discussions with A. Strominger. 
In our discussion above we have ignored the possibility that the D-branes leave the black hole since all our discussion concentrated on the hypermultiplet moduli space. As long as $R$ is not too small $R \geq \alpha^{\prime}$ D-brane emission will be suppressed because of the

change in entropy $\delta S \sim \pi \sqrt{\frac{Q_{5} n_{L, R}}{Q_{1}}}$ which is large in the region $r_{0}^{2}+r_{n}^{2} \gg 1$ corresponding to black holes with smooth horizons and small $\alpha^{\prime}$ corrections. The conclusion is that the D-brane system corresponding to smooth, big, classical black hole solutions always have large $n_{L}$ or $n_{R}$ so that D-brane emission is suppressed. Momentum emission (KK charge) could or could not be suppressed $\mathrm{\theta}$. Indeed, if the radius of $S_{1}$ is very large, then $n$ can be very large while we are still in the dilute gas region. In this case KK charge emission is not suppressed and the black hole, more properly a black string, will discharge. In the case that $R$ is small, say of the order of $\alpha^{\prime}$ then we could take all $Q_{1}, Q_{5}, n$ to be large in fixed proportions, then the charged emission will be suppressed. This can be intuitively understood by remembering that all charged particles would have large masses so they are not likely to be emitted. In fact, the suppression factor goes like $e^{\frac{-1}{\mathrm{RT}_{L}}}$ [13]. It is very important that in this case ( small $R$ ) the moduli space includes twisted sectors representing multiple windings, ensuring a small energy gap and the existence of the low energy excitations that account for the entropy. Notice that the total energy of the excitations on the moduli space is large but the temperature is small, due to the large number of degrees of freedom.

These reasons explaining why D-brane emission is suppressed also justify our restriction to the hypermultiplet moduli space in the D-brane analysis of sec. 4.

It seems that the best scenario for discussing the excitation and decay of an extremal black hole is the one with $R$ small and large $Q_{1}, Q_{5}, N$, since in this case the black hole does not fragment, it has a smooth geometry from the classical point of view and can be described by the D-brane moduli space as long as we are in the dilute gas and low energy regions (2.2).

\section{Information Loss}

We have shown above that starting with a D-brane system we can go to strong coupling and still continue having the same description at low energies. It includes back reaction and it keeps track of the black hole microstates. The description is unitary, the unitarity problem disappears when we use the full string theory.

7 The situation is U-duality asymmetric because we are insisting in $g \ll 1$ and $R \geq \alpha^{\prime}$. 
At the same time we have the traditional semiclassical description of the black hole. Since both descriptions pertain to the same physical object they should somehow agree. The semiclassical results are recovered when we trace over the black hole microstates provided by the D-brane description. It is important here that we are restricting to low energies (2.2), at low energies the black hole already looks like a pointlike system, so that replacing it by the D-brane moduli space theory just amounts to providing a description of the black hole states and their interactions with the outside world. This effective low energy theory is similar in spirit to the low energy description of the scattering of massless fermions off a magnetic monopole (Callan Rubakov effect) [34, where one replaces the monopole by a rotator sitting at the origin. It is clear that the low energy D-brane moduli space Hamiltonian is unitary, massive modes provide just small corrections. A big difference between the two descriptions is that the D-brane description keeps track of the black hole microstates. Only after tracing them out we get the usual thermodynamic description.

There is an interesting question: what exactly is the problem about the usual information loss argument in this case? The answer is not totally clear, it is an interesting problem. Hawking's thermal matrix [18] relies on tracing over the modes that go into the black hole, the D-brane picture suggests that one should think of these modes as part of the black hole excitations, so it is not reasonable to trace over them if one is keeping track of the changes in the black hole microstate as the radiation is emitted. There have been many suggestions in the literature of things that could be wrong like some non-locality of string theory at high relative boosts [35], the ideas of black hole complementarity [36], etc..

Even though this argument says that there is no information loss at low energies there could indeed be information loss at higher energies since the D-brane moduli space description is valid only at low energies. So the general question remains open but there is a corner (low energies) from which it seems eliminated.

It would be nice to extend these arguments to near extremal four dimensional black holes.

\section{Acknoledgements}

It is a pleasure to thank V. Balasubramanian, T. Banks, M. Douglas, D. Kabat, D. Lowe, S. Mathur, J. Polchinski, N. Seiberg, S. Shenker, A. Strominger and C. Vafa for discussions, valuable coments and suggestions. This work was supported in part by DOE grant DE-FG02-96ER40559. 


\section{References}

[1] A. Strominger and C. Vafa, "On the Microscopic Origin of the Bekenstein-Hawking Entropy”, Phys. Lett. B379 (1996) 99, hep-th/9601029.

[2] J.C. Breckenridge, R.C. Myers, A.W. Peet and C. Vafa, D-Branes and Spinning Black Holes", hep-th/9602065.

[3] J. Maldacena and A. Strominger, "Statistical Entropy of Four-Dimensional Extremal Black Holes", Phys. Rev. Lett. 77 (1996) 428, hep-th/9603060.

[4] C. Johnson, R. Khuri and R. Myers, Entropy of 4 D Extremal Black Holes", Phys. Lett. B378 (1996) 78, hep-th/9603061.

[5] G. Horowitz and A. Strominger, "Counting States of Near-extremal Black Holes ", Phys. Rev. Lett. 77 (1996) 2368, hep-th/9602051.

[6] C. Callan and J. Maldacena, "The D-brane approach to black hole quantum mechanics", Nucl. Phys. B 475 (1996) 645, hep-th/9602043.

[7] G. Horowitz, D. Lowe and J. Maldacena, "Statistical Entropy of Nonextremal Four Dimensional Black Holes and U-duality", Phys. Rev. Lett. 77 (1996) 430, hepth/9603195.

[8] J. C. Breckenridge, D. A. Lowe, R. C. Myers, A. W. Peet, A. Strominger and C. Vafa, "Macroscopic and Microscopic Entropy of Near-Extremal Spinning Black Holes", Phys. Lett. B381 (1996) 423, hep-th/9603078.

[9] I. Klebanov and A. Tseytlin, "Intersecting M-Branes as Four Dimensional Black Holes" Nucl. Phys. B475 (1996) 179, hep-th/9604166; V. Balasubramanian and F. Larsen, "On D-Branes and Black Holes in Four Dimensions", hep-th/9604189.

[10] S. Dhar, G. Mandal and S. Wadia, "Absorption vs. Decay of Black Holes in String Theory and T-symmetry", hep-th/9605234.

[11] S. Das and S. Mathur, "Comparing Decay Rates for Black Holes and D-branes", hepth/9606185; "Interactions Involving D-branes", hep-th/9607149.

[12] S. Gubser and I. Klebanov, "Emission of Charged Particles from Four- and Fivedimensional Black Holes", hep-th/9608108.

[13] J. Maldacena and A. Strominger, "Black Hole Greybody Factor and D-brane Spectroscopy", hep-th/9609026.

[14] S. Gubser and I. Klebanov, "Four Dimensional Grey Body Factors and the Effective String", hep-th/9609076.

[15] C. Callan, S. Gubser, I. Klebanov and A. Tseytlin, "Absorption of Fixed Scalars and the D-Brane Approach to Black Holes", hep-th/9610172.

[16] G. Horowitz, J. Maldacena and A. Strominger, "Nonextremal Black Hole Microstates and U-duality", Phys. Lett. B383 (1996) 151, hep-th/9603109.

[17] S. Hawking, "Particle Creation by Black Holes", Comunn. Math. Phys. 43 (1975) 199. 
[18] S. W. Hawking, Breakdown of Predictability in Gravitational Collapse", Phys. Rev. D14 (1976) 2460.

[19] N. Seiberg and E. Witten, "Gauge Dynamics and Compactification to Three Dimensions", hep-th/9607163.

[20] M. Douglas, Branes within Branes, hep-th/9512077.

[21] M. Bershadsky, V. Sadov and C. Vafa, "D-Strings on D-Manifolds", Nucl. Phys. B463 (1996) 398, hep-th/9511222; C. Vafa, "Instantons on D-Branes", Nucl. Phys. B463 (1996) 435, hep-th/9512078.

[22] R. Dijkgraaf, E. Verlinde and H. Verlinde, "Counting Dyons in N=4 String Theory", hep-th/9607026.

[23] R. Dijkgraaf, G, Moore, E. Verlinde and H. Verlinde, "Elliptic Genera of Symmetric Products and Second Quantized Strings", hep-th/9608096.

[24] B. de Wit, P. Lauwers and A. Van Proeyen, "Lagrangians of N=2 Supergravity-Matter systems", Nucl. Phys. B255 (1983) 569.

[25] K. Intrilligator and N. Seiberg, "Mirror Symmetry in Three Dimensional Gauge Theories", hep-th/9607207.

[26] S. Gates, C. Hull and M. Roček, "Twisted Multiplets and New Supersymmetric NonLinear $\sigma$-Models", Nucl. Phys. B248 (1984) 157.

[27] L. Alvarez-Gaume and D. Freedman, "Geometrical Structure and Ultraviolet Finitness in the Supersymmetric Sigma Model', Comm. Math. Phys. 80 (1981) 443, and references therein.

[28] J. Maldacena, "Black Holes in String Theory", Ph.D. thesis, Princeton University 1996, hep-th/9608235.

[29] N. Seiberg, "Naturalness vs. Supersymmetric Non-renormalization Theorems", Phys. Lett. B318 (1993) 469, hep-th/9309335; "Power of Holomorphy- Exact Results in $4 D$ Supersymmetric Field Theories", PASCOS 1994, 357-369 (QCD161:I69:1994), hepth/9408013.

[30] M. Douglas, D. Kabat, P. Pouliot and S. Shenker, "D-branes and Short Distances in String Theory", hep-th/9608024.

[31] T. Banks, W. Fischler, S. Shenker and L. Susskind, "M Theory As A Matrix Model: A Conjecture", hep-th/9610043.

[32] T. Banks, M. Douglas, J. Polchinski, S. Shenker and A. Strominger, private comunication.

[33] J. Maldacena and L. Susskind, "D-branes and Fat Black Holes ", Nucl. Phys. B475 (1996) 679, hep-th/9604042.

[34] J. Polchinski, "Monopole Catalysis: The Fermion Rotor System", Nucl. Phys. B242 (1984) 345; Rubakov, Nucl. Phys. B203 (1982) 311; C. Callan, Phys. Rev. D25 (1982) 2141; D26 (1982) 2058; Nucl. Phys. B212 (1983) 391. 
[35] D. Lowe, J. Polchinski, L. Susskind, L. Thorlacius and J. Uglum, "Black Hole Complementarity vs. Locality", Phys.Rev. D52 (1995) 6997, hep-th/9506138.

[36] G. 't Hooft, "The Black Hole Interpretation of String theory", Nucl.Phys B 335 (1990) 138; G. 't Hooft, The Scattering Matrix Approach for the Quantum Black Hole: An Overview" Int. J. Mod. Phys. A11 (1996)4623, gr-qc/9607022; Y. Kiem, H. Verlinde and E. Verlinde, "Black Hole Horizons and Complementarity", Phys.Rev.D52, (1995) 7053 hep-th/9502074. 